\title{
Norois
}

Environnement, aménagement, société

\section{André Lespagnol et Matthieu Leprince, 2016. Les mutations de l'enseignement supérieur et de la recherche en Bretagne (1945-2015). Déploiement territorial, diversification et essais de structuration} Raymonde Séchet

\section{(2) OpenEdition}

\section{Journals}

Édition électronique

URL : http://journals.openedition.org/norois/6132

DOI : $10.4000 /$ norois. 6132

ISBN : 978-2-7535-6537-1

ISSN : $1760-8546$

Éditeur

Presses universitaires de Rennes

\section{Édition imprimée}

Date de publication : 2 novembre 2017

Pagination : 127-130

ISBN : 978-2-7535-6465-7

ISSN : 0029-182X

Référence électronique

Raymonde Séchet, «André Lespagnol et Matthieu Leprince, 2016. Les mutations de l'enseignement supérieur et de la recherche en Bretagne (1945-2015). Déploiement territorial, diversification et essais de structuration », Norois [En ligne], 243 | 2017, mis en ligne le 20 septembre 2019, consulté le 15 mars 2021. URL : http://journals.openedition.org/norois/6132 ; DOI : https://doi.org/10.4000/norois.6132 


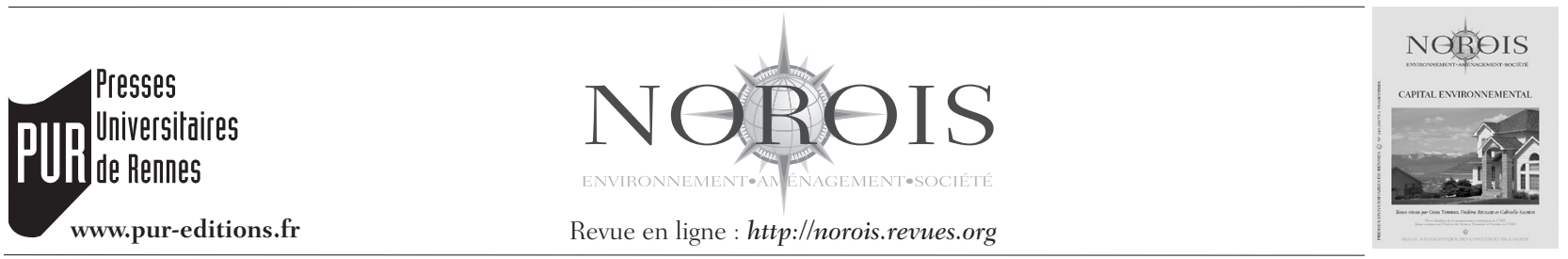

COMPTE RENDU BIBLIOGRAPHiQue

\begin{abstract}
André Lespagnol et Matthieu Leprince, 2016. Les mutations de l'enseignement supérieur et de la recherche en Bretagne (1945-2015). Déploiement territorial, diversification et essais de structuration, Rennes, PUR, coll. «Espace et Territoires », 482 p.
\end{abstract}

L'annonce de l'échec du projet d'I-Site ${ }^{1}$ rennais, après celui de Brest au premier tour de la sélection, la présence de Rennes, avec Grenoble et Toulouse, dans le trio de tête des villes préférées des étudiants ${ }^{2}$ : ces deux informations de février 2017 , certes d'inégale importance on le concédera, illustrent bien la situation actuelle de l'enseignement supérieur en Bretagne, ses forces et ses faiblesses, ainsi que les tensions qui le traversent. Elles sont à décoder au regard de la construction du dispositif régional d'enseignement supérieur qu'analyse l'ouvrage Les mutations de l'enseignement supérieur et de la recherche en Bretagne (19452015). Déploiement territorial, diversification et essais de structuration coordonné par André Lespagnol et Matthieu Leprince.

Pluridisciplinaire et réunissant des textes de chercheurs et d'anciens acteurs de l'enseignement supérieur ${ }^{3}$, l'ouvrage prolonge le programme de

1. Les I-SITE sont l'un des deux volets de l'action IDEX/I-SITE engagée en 2014 dans le cadre du second programme d'Investissements d'avenir (PIA2). Ils s'appuient sur des potentiels scientifiques thématiques reconnus à l'échelle internationale et sont supposés avoir un effet d'entraînement sur le tissu local. Le jury international a reconnu la richesse de la recherche rennaise mais a émis des critiques sur les aspects relatifs à la gouvernance du projet.

2. Classement annuel établi par L'Étudiant. Pour 2016-2017 : [http://www. letudiant.fr/palmares/liste-profils/palmares-des-villes-etudiantes/palmaresdes-villes/home.html].

3. L'auteure de cette note de lecture fait partie de ces acteurs. Elle a été directrice de l'UFR «Sciences sociales» de l'université Rennes 2 de 1998 à 2002, puis directrice de l'UMR ESO de 2002 à 201 l, avant d'être élue vice-présidente en charge de la recherche de l'université Rennes 2 recherche HESRIB « Histoire du développement et de la structuration de l'enseignement supérieur, de la recherche et de l'innovation en Bretagne depuis 1945 » et le colloque « Du pôle aux réseaux. Autour de la construction d'un système d'enseignement supérieur et de recherche en Bretagne (1945-2015) » qui s'est tenu à Rennes en juin 2015. Les 22 textes sont regroupés en 4 parties d'inégale ampleur portant sur le déploiement territorial, la diversification des filières et des structures, l'essor de la recherche, les difficultés des efforts de structuration régionale. La lecture d'ensemble révèle combien certains acteurs (dont le recteur Henri Le Moal et Alice Picard qui deviendra la ministre Alice Saunier-Seïté $)^{4}$ ont joué un rôle majeur dans la mise en œuvre d'un système régional de l'enseignement supérieur et de la recherche qui ne saurait se comprendre uniquement par le réseau urbain régional.

Le déploiement territorial de l'enseignement supérieur en Bretagne s'est fait à partir de la seule université de Rennes, d'abord vers Brest, puis vers les villes

\footnotetext{
(janvier 2011 à janvier 2015), et, à ce titre, impliquée dans plusieurs des débats relatifs à l'enseignement supérieur et la recherche en Bretagne.

4. Professeur de chimie, doven de la Faculté des sciences de Rennes en 1958, Henri Le Moal a été nommé recteur de l’Académie de Rennes en 1960. Il le reste jusqu'en 1970, et est donc en fonction lors de la partition qui aboutit à la création des universités de Rennes 1 et Rennes 2. La géographe Alice Saunier a été secrétaire d'État aux universités dans les gouvernements de Jacques Chirac et Raymond Barre (janvier 1976mars 1977) puis ministre des Universités dans les gouvernements de Raymond Barre (mars 1977 à mai 1981).
} 


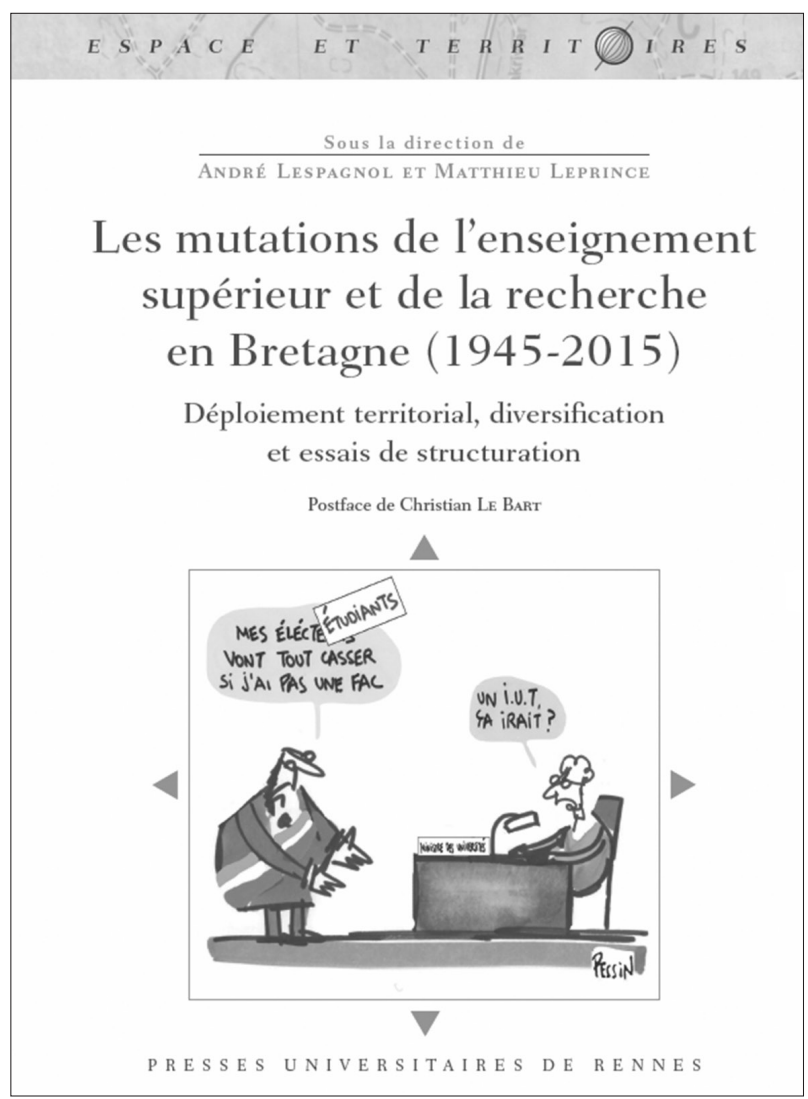

moyennes qui ont su tirer parti du soutien national à la création de départements d'IUT ou d'opportunités en matière de recherche. En même temps qu'elle se déployait dans l'espace régional, l'université de Rennes a évolué. Son éclatement (A. Lespagnol) trouve peut-être ses prémices dans l'échec du projet de grand campus unique de Rennes (1945-1954). La première faille identifiée dans cette utopie (M. Leprince) a été la construction d'un bâtiment neuf en centre-ville par la faculté de droit qui a fait preuve d'opportunisme pour mobiliser des financements d'État. La loi Faure du 12 novembre 1968 a débouché en Bretagne sur l'autonomisation du site brestois et la création de l'université de Bretagne occidentale, et sur la partition du site rennais entre l'université de Rennes 1 réunissant 3 des 4 anciennes facultés, et la future université Rennes 2 réduite à la faculté des Lettres, «mise au coin » selon son doyen Marache, et, qui plus est, amputée de la philosophie. Cette partition allait déboucher sur des relations compliquées entre les deux universités rennaises et poser des problèmes de cohérence qui ne sont pas encore totalement résolus.
La création de filières AES dans les deux universités rennaises en 1973 s'inscrit dans l'encouragement à la mise en place de formations ouvertes vers les milieux professionnels par la loi Faure. Elle illustre les rapports de force en présence et la différenciation des enjeux pour chacune des universités (C. Dumoulin, A. Filhon). Les orientations sont différentes : plus économique à Rennes 1 , plus orientée vers les sciences sociales et accordant plus de place aux stages professionnels à Rennes 2. La diversification des formations ayant été une nécessité pour Rennes 2, la Présidence a apporté un soutien fort à la création d'une licence AES (ainsi que MASS et LEA). À Rennes 1, le projet a été soutenu par de jeunes enseignants d'économie dont Yves Morvan et le président de l'université Claude-Champaud, mais sans le soutien de la majorité des enseignants d'économie, restés attachés au modèle facultaire. Les rapports de force entre les deux universités se sont également exprimés autour du rattachement universitaire des formations en éducation physique et sportive, Rennes 1 arguant des liens nécessaires avec la santé, Rennes 2 mettant en avant les besoins d'une solide formation en psychologie (J. Fuchs et al.).

Le déploiement territorial par délocalisation de formations de premier cycle a accompagné la forte croissance des effectifs étudiants, notamment pour des filières généralistes comme le droit. (D. Gadbin). Les édiles des villes moyennes n'ont toutefois pas fait preuve de la même capacité de mobilisation. Enfermés dans leur idéologie d'adéquation avec le tissu économique local, ceux des années 1970 à Saint-Brieuc n'ont rien fait pour favoriser l'implantation de formations universitaires et ont préféré soutenir la création de sections de techniciens supérieurs dans les lycées (P. Harismendy). À l'inverse, à Quimper, la convergence entre les objectifs des acteurs locaux, politiques et enseignants, et l'entregent d'Alice Picard ont abouti à la création d'un pôle universitaire secondaire (J.-L. Le Cam). La création de l'université de Bretagne-Sud découle quant à elle de la "volonté collective du Morbihan ", c'est-à-dire de la pugnacité des édiles locaux qui ont su faire abstraction de leurs divergences politiques pour obtenir leur université (L. Métayer-Noël).

À la diversification des formations universitaires s'est ajoutée celle des structures de recherche. Le dernier élément marquant est la création de l'ENS de Rennes sur l'atypique Campus de Ker Lann, né 
de la volonté du président du Conseil général d’Illeet-Vilaine, Pierre Méhaignerie (A. Charraud). Cette création, promise par la ministre Valérie Pécresse en 2009, abandonnée par son successeur Laurent Wauquiez en 2012, est finalement annoncée à la fin de la même année par Geneviève Fioraso, sans doute éclairée par son collègue breton du gouvernement, Jean-Yves Le Drian (F. Le Bot). Des épisodes précédents, on retiendra les vagues successives de création d'écoles d'ingénieurs dans les domaines de l'électronique et de l'informatique après l'impulsion initiale de l'État dans les années 1960. Écoles, universités, organismes de recherche ont coopéré pour structurer un ensemble qui fonctionne en un réseau cohérent implanté à Rennes, Brest, ainsi que Nantes, mais aussi Lannion, Lorient, Vannes, et qui a permis à la Bretagne de rivaliser avec les grands pôles technologiques nationaux (J.-Y. Merrien, P. Dupuis).

L'annonce de la création du CNEXO (Centre national pour l'exploitation des océans) en 1966 a exacerbé la concurrence entre pôles régionaux de recherche en portant un coup final au projet rennais d'Institut des Sciences de la mer (A. Lespagnol), mais elle surtout a été le point de départ de l'émergence d'un pôle majeur de recherche en sciences de la mer à Brest. Né de la volonté du général de Gaulle de rester dans la course « pour l'accaparement de la mer » (Ferrière, Laubé), le CNEXO vient compléter et en même temps concurrencer l'ISTPM (Institut des sciences et techniques des pêches maritimes) déjà présent sur le littoral breton (G. Chatry). L'idée de fusion émerge donc rapidement. Elle est finalement décidée en conseil des ministres en 1982 et se traduit par la création de l'IFREMER placée sous la double tutelle du ministre de la Recherche et de celui de la Mer.

Des institutions de recherche ont aussi été localisées dans des villes plus modestes que les deux métropoles régionales (J. Tallec). C'est au nom des besoins de l'agroalimentaire que la décision d'implanter le laboratoire d'études et de recherches sur les médicaments vétérinaires, devenu depuis l'ANSES (agence nationale de sécurité sanitaire, de l'alimentation, de l'environnement et du travail) à Fougères est prise en 1975, par ailleurs confrontée à une grave crise industrielle et alors que le maire de la ville, Michel Cointat, a été ministre de l'agriculture, et que l'ADRIA (Association pour le développement, la recherche et l'innovation agroa- limentaire) spécialisée en microbiologie a été créée à Quimper en 1971. Dans les deux cas, mobilisation des milieux professionnels régionaux, intercessions étatiques, présence d'édiles locaux influents ont combiné leurs effets. Toutefois, les relations entretenues entre leurs activités scientifiques et le territoire d'implantation sont complexes et la contribution au développement local est moins claire que pour des secteurs réunissant de multiples structures dans des interactions recherche, formation, innovation.

Les impulsions étatiques se sont poursuivies dans les années 1990, dans le cadre du Plan U2000 pour répondre à la forte croissance des effectifs (P. Mériot; $\mathrm{M}$. André) tout en soutenant le développement de la recherche universitaire (Y. Morvan). U2000 a consacré le pouvoir de la Région en matière de recherche, témoigné de l'intervention croissante des deux métropoles, Rennes et Brest, pour réclamer des chercheurs, et exigé des universités qu'elles soient des instruments de l'aménagement des territoires.

Outre ceux de l'IFREMER, de l'ANSES, de l'ADRIA, la politique de rééquilibrage Parisprovince, Est-Ouest de la France conduite dans les années 1960 et au tournant des années 1990 a favorisé la venue en Bretagne de chercheurs de l'INRA, de l'INRIA, du CNRS, et a ainsi permis « un certain rééquilibrage à défaut d'un rééquilibrage certain » (G. Baudelle) des effectifs des organismes de recherche. Mais force est de constater que les politiques de structuration de la recherche conduites depuis une dizaine d'années avantagent les grands pôles et creusent les inégalités sur le territoire national, déchirant le couple enseignement supérieur et aménagement du territoire (D. Le Couédic). Le système régional d'enseignement supérieur et de recherche, confronté aux craintes et frilosités de certains de ses acteurs, à ses concurrences internes et ses occasions ratées, aux incertitudes quant à sa gouvernance (C. Soldano), n'a en effet pas été en mesure de convaincre les jurys internationaux de son « excellence».

Lecteurs et lectrices, en fonction de leurs positions dans le monde académique ou en dehors, pourront regretter telle ou telle absence : les facultés de médecine, l'agronomie, l'environnement, la formation des maîtres devenue celle des enseignants, etc. Mais au final l'ouvrage coordonné par André Lespagnol et Matthieu Leprince, va bien au-delà de l'analyse de la construction d'un système régio- 
nal d'enseignement supérieur. Par le décryptage des réalisations, des réussites, des inachèvements, il met en évidence la nécessité pour les acteurs de l'enseignement supérieur et de la recherche et pour les décideurs des villes, des régions, de l'État d'agir dans la même direction et avec les mêmes objectifs - l'intérêt des étudiants, des territoires, des sociétés dans leurs différentes composantes. Cela tout en restant à l'écoute de ceux qui font l'université et la recherche pour que les priorités des uns soient celles de tous. En cela, il a une portée qui dépasse le cadre de la Bretagne.

Raymonde SÉCHET 